\title{
GAUSSIAN RANDOM BAND MATRICES AND OPERATOR-VALUED FREE PROBABILITY THEORY
}

\author{
DIMITRI SHLYAKHTENKO \\ Department of Mathematics, University of California \\ Berkeley, California 94720, U.S.A. \\ E-mail: shlyakht@member.ams.org \\ Current address: Department of Mathematics, UCLA, Los Angeles, California 90095, U.S.A. \\ E-mail: shlyakht@sonia.math.ucla.edu
}

1. Gaussian random band matrices. Let $g_{i j}^{k}, 1 \leq i, j \leq n, k=1, \ldots, r$ be independent centered complex Gaussian random variables of covariance $\frac{1}{n}\left(1+\delta_{i j}\right)$ (i.e., the expectation $\left.E\left(g_{i j}^{k} \bar{g}_{i j}^{k}\right)=\frac{1}{n}\left(1+\delta_{i j}\right)\right)$, such that $g_{i j}^{k}=\bar{g}_{j i}^{k}$. Let for each $i, j, k, \sigma_{n}(i, j ; k)$ be a positive real number, such that $\sigma_{n}(i, j ; k)=\sigma_{n}(j, i ; k)$. Consider the $r$-tuple of $n \times n$ matrices $G_{n}(k)=\left(\sigma_{n}(i, j ; k) g_{i j}^{k}\right)_{i j}, k=1, \ldots, r$. The family $\left\{G_{n}(k)\right\}_{k}$ is called a family of independent Gaussian random band matrices. As an example, assume that $\sigma_{n}(i, j ; k)=1$ if $|i-j|<c n$, and is zero otherwise. In this case all entries of $G_{n}(k)$ are zero outside a band around the diagonal; this is the reason such matrices are called band matrices. In the case that $\sigma_{n}(i, j ; k)$ is identically 1 one recovers the so-called Gaussian Unitary Ensemble, see e.g. [9].

1.1. Let $R_{n}=*-\operatorname{Alg}\left(g_{i j}^{k}, 1 \leq i, j \leq n, 1 \leq k \leq r\right)$ be the $*$-algebra generated by the entries of $G_{n}(k)$. Let $A_{n}=R_{n} \otimes M_{n \times n}$ be $n \times n$ matrices over $R_{n}$; hence $G_{n}(k) \in A_{n}$. Note that $1 \otimes M_{n \times n} \subset A_{n}$ as matrices with scalar entries. Let $\Delta_{n} \subset 1 \otimes M_{n \times n} \subset A_{n}$ be the algebra of diagonal matrices with complex entries. The map $E_{n}$ taking expectation of each diagonal entry of a matrix in $A_{n}$ and replacing off-diagonal entries by zeros defines a conditional expectation from $A_{n}$ onto $\Delta_{n}$, i.e, $E_{n}$ satisfies $E_{n}\left(d x d^{\prime}\right)=d E(x) d^{\prime}$ for all $d, d^{\prime} \in \Delta_{n}, x \in A_{n}$, and $E_{n}(d)=d, d \in \Delta_{n}$. Composing $E_{n}$ with the normalized trace $\tau_{n}=\frac{1}{n} \operatorname{Tr}$ gives a linear functional $\phi_{n}$ on the non-commutative algebra $A_{n}$, with $\phi_{n}(1)=1$. Therefore, $\left(A_{n}, \phi_{n}\right)$ is a non-commutative probability space [26]; and elements of $A_{n}$ (e.g., the matrices $\left.G_{n}(k)\right)$ are non-commutative random variables.

1991 Mathematics Subject Classification: Primary 46L50; Secondary 60H25.

This material is based upon work supported under a National Science Foundation Graduate Research fellowship.

The paper is in final form and no version of it will be published elsewhere. 
1.2. Using $\phi_{n}$ one can define the joint distribution $\mu_{f_{1}, \ldots, f_{k}}$ of any $k$-tuple of elements in $A_{n}$. The joint distribution $\mu_{f_{1}, \ldots, f_{k}}$ is a linear functional from the space $\mathbf{C}\left[X_{1}, \ldots, X_{k}\right]$ of polynomials in $k$ non-commutative indeterminants with coefficients in $\mathbf{C}$, defined on monomials by

$$
\mu_{f_{1}, \ldots, f_{k}}\left(X_{i_{1}} \ldots X_{i_{n}}\right)=\phi\left(f_{i_{1}} \ldots f_{i_{n}}\right) .
$$

Since the elements $G_{n}(k)$ are self-adjoint, the functionals $\mu_{G_{n}(k)}$ can be extended from complex polynomials in one variable to functions on the real line, thus defining probability measures. The measure $\mu_{G_{n}(k)}$ is called the distribution of $G_{n}(k)$.

1.3. Let $\Delta_{n} \subset 1 \otimes M_{n \times n} \subset A_{n}$ be the algebra of diagonal complex-valued matrices, and let $d_{n}=d_{n}^{*} \in \Delta_{n}$ be a choice of a diagonal matrix. For a variety of applications, one is interested in the asymptotics as $n \rightarrow \infty$ of the measures $\mu_{d_{n}+G_{n}(1)}$, under assumptions that $d_{n}$ converge in some sense. For example, the random matrix $H_{n}=d_{n}+G_{n}(1)$ can be used as a Hamiltonian operator, where $d_{n}$ models a "deterministic" interaction, and $G_{n}(k)$ models a "random" interaction. Since the scales of deterministic and random interactions may be different, it may be necessary to arrange for the off-diagonal entries of $G_{n}(1)$ to decay sufficiently fast; this can be accomplished by the appropriate choice of $\sigma_{n}(i, j ; 1)$. For a sample physical application, see [1].

2. Asymptotic scalar-valued freeness. The limits of the distribution of $H_{n}$ have been computed by ad hoc methods before (see e.g. [5, 4, 10, 3, 1]).

However, in the case $\sigma=1$ identically, Voiculescu has connected asymptotic behavior of matrices $d_{n}, G_{n}(1), \ldots, G_{n}(r)$ with his free probability theory, and has given analytical methods for computation of the spectrum of $H_{n}$ (see [26, 21, 25, 24, 22, 23]). Recall that if $(A, \phi)$ is a non-commutative probability space and $A_{i}, i \in I$ are unital subalgebras of $A$, then the algebras $A_{i}$ are free, if

$$
\phi\left(x_{1} \ldots x_{p}\right)=0
$$

whenever $x_{j} \in A_{i(j)}, \phi\left(x_{j}\right)=0$ for all $j$, and $i(j) \neq i(j+1), j=1, \ldots, p-1$. Elements $f_{1}, \ldots, f_{n}$ of $A$ are free, if the algebras $\operatorname{Alg}\left(f_{1}\right), \ldots, \operatorname{Alg}\left(f_{n}\right)$ are free.

2.1. Theorem (Voiculescu, [24]). Let $G_{n}(k), d_{n}$ be as before, and assume $\sigma_{n}(i, j ; k)$ $=1, \sup _{n}\left\|d_{n}\right\|<\infty$, and $\mu_{d_{n}}$ converges weakly as $n \rightarrow \infty$. Then there exists a noncommutative probability space $(A, \phi)$, and elements $d, G(1), \ldots, G(r) \in A$, such that in the weak topology,

$$
\lim _{n \rightarrow \infty} \mu_{d_{n}, G_{n}(1), \ldots, G_{n}(r)}=\mu_{d, G(1), \ldots, G(r)} .
$$

Furthermore, the family $(d, G(1), \ldots, G(r))$ satisfies:

(1) $d, G(1), \ldots, G(r)$ are free,

(2) $\mu_{G(k)}$ is the linear functional of integration against the semicircular measure $\frac{1}{\pi}(1-$ $\left.t^{2}\right)^{1 / 2} d t$.

So in the case $\sigma=1$ identically, the problem of computation of $\lim _{n} \mu_{H_{n}}$ becomes the problem of computing $\mu_{d+Z}$, where $\mu_{d}=\lim _{n} \mu_{d_{n}}, \mu_{Z}$ is the semicircular measure, and $d$ and $Z$ are free. By analogy with the classical case, Voiculescu defined $\mu_{d+Z}$ to be the free additive convolution of $\mu_{d}$ and $\mu_{Z}$. The computation of this convolution, and so of 
$\mu_{d+Z}$, can be done using the analytical machinery of the $R$-transform; see below and also $[26,22]$.

2.2. It is natural to ask whether a similar theorem holds for general $\sigma$. The answer is easily seen to be no: observe that $G_{n}(k)$ satisfy

$$
\mu_{\frac{1}{\sqrt{p}}\left(G_{n}(1)+\ldots+G_{n}(p)\right)}=\mu_{G_{n}(1)},
$$

since a sum of independent Gaussian random variables is Gaussian. Hence if in the limit $n \rightarrow \infty, G_{n}(1), \ldots, G_{n}(p)$ become free, and have limit distributions $\mu_{G(1), \ldots, G(p)}$, then one also has $\mu_{\frac{1}{\sqrt{p}}(G(1)+\ldots+G(p))}=\mu_{G(1)}$. But by the free central limit theorem of Voiculescu (see $[26,21])$,

$$
\lim _{p \rightarrow \infty} \mu_{\frac{1}{\sqrt{p}}(G(1)+\ldots+G(p))}
$$

is semicircular, so that $\mu_{G(1)}$ is semicircular as well. On the other hand, it is clear that for certain choices of $\sigma, G_{n}(k)$ will have a limit distribution that is not semicircular; for example, take $\sigma_{n}(i, j ; k)=1$ if $1 \leq i, j \leq n / 2$, and zero otherwise. Then the limit distribution of $\mu_{G_{n}(1)}$ as $n \rightarrow \infty$ is $\frac{1}{2 \pi}\left(1-t^{2}\right)^{1 / 2} d t+\frac{1}{2} \delta_{0}$, where $\delta_{0}$ is a point mass at zero.

3. Asymptotic operator-valued freeness. It turns out that one can still recover an analog of Theorem 2.1 about asymptotic freeness of random matrices $G_{n}(k)$, at the price of using operator-valued free probability theory.

3.1. A triple $(B \subset A, E: A \rightarrow B)$ is called a non-commutative B-valued probability space ([26]), if $B \subset A$ is a unital subalgebra, and $E$ is a conditional expectation, i.e., $E\left(b a b^{\prime}\right)=b E(a) b^{\prime}$, for all $b, b^{\prime} \in B, a \in A$, and $E(b)=b$, for all $b \in B$. We sometimes require that $A$ and $B$ are $C^{*}$-algebras, and $E: A \rightarrow B$ is positive (i.e., $E\left(x^{*} x\right) \geq 0$ for all $x \in A)$; in this case we call the $B$-probability space $A$ a $C^{*}-B$-probability space. Note that a non-commutative $\mathbf{C}$-valued probability space is just a non-commutative probability space. More generally, if $(B \subset M, E: M \rightarrow B)$ is a $B$-valued probability space and $\phi: B \rightarrow \mathbf{C}$ is a unital linear functional, then $(M, \phi \circ E)$ is a non-commutative probability space.

3.2. Elements of a non-commutative $B$-valued probability space are called $B$-valued random variables. Given a family $f_{1}, \ldots, f_{k}$ of $B$-valued random variables, its joint $B$ distribution $\mu_{f_{1}, \ldots, f_{k} ; B}$ is defined as a $B$-linear map from polynomials in $k$ non-commuting indeterminates with coefficients in $B, B\left[X_{1}, \ldots, X_{n}\right]$, into $B$, given by

$$
\mu_{f_{1}, \ldots, f_{k} ; B}\left(b_{0} X_{i_{1}} b_{1} \ldots X_{i_{n}} b_{n}\right)=E\left(b_{0} f_{i_{1}} b_{1} \ldots f_{i_{n}} b_{n}\right) .
$$

In particular, the maps $b \mapsto E\left(f_{i} b f_{j}\right)$ are called the covariance of the family $f_{1}, \ldots, f_{k}$.

3.3. Let the algebra of random matrices $A_{n}$, the algebra of diagonal matrices with scalar entries $\Delta_{n}$, and $E_{n}: A_{n} \rightarrow \Delta_{n}$ be as in $\S 1.1$. Instead of viewing $A_{n}$ as a noncommutative scalar-valued probability space, we can view the triple $\left(\Delta_{n} \subset A_{n}, E_{n}\right.$ : $A_{n} \rightarrow \Delta_{n}$ ) as a $\Delta_{n}$-valued non-commutative probability space, and elements of $A_{n}$ as $\Delta_{n^{-}}$ valued random variables. Our aim is to compute the (scalar-valued) limit distribution of the family $\left(d_{n}, G_{n}(1), \ldots, G_{n}(r)\right)$. But this information is encoded in the $\Delta_{n}$-distribution 
of $G_{n}(1), \ldots, G_{n}(r)$. Indeed, knowledge of the joint distribution of $\left(d_{n}, G_{n}(1), \ldots, G_{n}(r)\right)$ is equivalent to knowing the numbers

$$
\phi_{n}\left(d_{n}^{j_{1}} G_{n}\left(k_{1}\right) d_{n}^{j_{1}} G_{n}\left(k_{2}\right) \ldots G_{n}\left(k_{p}\right) d_{n}^{j_{p+1}}\right) .
$$

But $\phi_{n}(x)=\tau_{n}\left(E_{n}(x)\right)$, so we know the number in (3.1) if we know the value of

$$
E_{n}\left(d_{n}^{j_{1}} G_{n}\left(k_{1}\right) d_{n}^{j_{1}} G_{n}\left(k_{2}\right) \ldots G_{n}\left(k_{p}\right) d_{n}^{j_{p+1}}\right)
$$

i.e., if we know the $\Delta_{n}$-distribution of the family $G_{n}(1), \ldots, G_{n}(r)$.

3.4. Given a $B$-valued probability space $(B \subset A, E: A \rightarrow B)$, a family of subalgebras $B \subset A_{i} \subset A$ is said to be free with amalgamation over $B$ ([26]), if

$$
E\left(x_{1} \ldots x_{p}\right)=0
$$

whenever $x_{j} \in A_{i(j)}, E\left(x_{j}\right)=0$, for all $j$, and $i(j) \neq i(j+1), j=1, \ldots, p-1$. A family $\left(f_{1}, \ldots, f_{r}\right)$ of $B$-valued random variables in $A$ are free with amalgamation over $B$, if the family of subalgebras $\operatorname{Alg}\left(f_{i}, B\right), i=1, \ldots, r$, are free with amalgamation over $B$.

3.5. In order to describe the limit distribution of the family $G_{n}(k)$ we need to introduce the operator valued semicircular family of Speicher ([20]). Let $B$ be a $C^{*}$-algebra, and $\eta_{i j}: B \rightarrow B, 1 \leq i, j \leq r$ be linear maps, such that the associated map $\eta: B \rightarrow B \otimes M_{r \times r}$ is completely positive. Then there exists a Hilbert $(B, B)$-bimodule $H$ (i.e., a $(B, B)$ bimodule endowed with a $B$-valued inner product $\langle\cdot, \cdot\rangle$, and which is complete in the resulting norm, see e.g. [2] for details) and vectors $\xi_{i} \in H, 1 \leq i \leq r$, satisfying

$$
\left\langle\xi_{i}, b \xi_{j}\right\rangle=\eta_{i j}(b), \quad b \in B .
$$

Consider the full Fock space

$$
F_{B}(H)=B \oplus H \oplus\left(H \otimes_{B} H\right) \oplus \ldots .
$$

Here $\otimes_{B}$ refers to the relative tensor product over $B$. Consider the free creation operators

$$
L_{i}: F(H) \ni \xi \mapsto \xi_{i} \otimes \xi \in F(H), \quad 1 \leq i \leq r
$$

(cf. [11]). Then one has the relation

$$
L_{i}^{*} b L_{j}=\eta_{i j}(b), \quad b \in B,
$$

where $b \in B$ is viewed as an operator acting on the left on $F_{B}(H)$. Let $E: C^{*}\left(B, L_{i}\right.$ : $1 \leq i \leq r) \rightarrow B$ be the map defined by $E(b)=b, b \in B$, and $E(W)=0$ if $W$ is a word in elements from $B$ and $L_{i}, L_{j}^{*}, 1 \leq i, j \leq r$, which cannot be reduced to an element of $B$ using the relation (3.2). Let $P$ be the orthogonal projection from $F(H)$ onto $B \subset F(H)$. Then $P$ commutes with the left action of $B$ and $E(x) P=P x P$ for all $x \in C^{*}\left(B, L_{i}: 1 \leq i \leq r\right)$. From this it follows that $E$ is well-defined and is a conditional expectation (and is in fact positive, $E\left(x^{*} x\right) \geq 0$ ). Let $Z_{i}=L_{i}+L_{i}^{*}$. The family of $B$-valued random variables $Z_{i}$ in the $B$-probability space $\left(B \subset C^{*}\left(B, Z_{i}: 1 \leq i \leq r\right), E\right)$ is called a $B$-valued semicircular family of covariance $\eta$. The name comes from the fact that the covariance (see $\S 3.2) E\left(Z_{i} b Z_{j}\right)=\eta_{i j}(b)$ for $b \in B$.

3.6. TheOREM (Speicher, [20]). Let $Z_{i}$ be a B-semicircular family of covariance $\eta$. Then $Z_{i}$ are free with amalgamation over $B$ if and only if $\eta_{i j}=0$ for $i \neq j$. 
Speicher proved the following Theorem, which is a central limit theorem for $B$-valued random variables, free with amalgamation over $B$. It shows that $B$-valued semicircular variables play the role of Gaussian families in $B$-valued free probability theory. This Theorem is stated in his paper for the case of identically distributed variables, but is true more generally:

3.7. TheOREM (Speicher, [20]). Let $B$ be a $C^{*}$-algebra. Let $X_{n}(1), \ldots, X_{n}(r)$ be $B$ valued random variables in a $C^{*}-B$-probability space $(B \subset A, E: A \rightarrow B)$, and assume that the covariances $\eta_{i j}^{(n)}(b)=E\left(X_{n}(i) b X_{n}(j)\right), b \in B$, converge pointwise in norm to bounded maps $\eta_{i j}: B \rightarrow B$. Assume further that $E\left(X_{n}(k)\right)=0,1 \leq k \leq r$, and the maps

$$
\left(b_{0}, b_{1}, \ldots, b_{p}\right) \mapsto E\left(b_{0} X_{n}\left(i_{1}\right) b_{1} \ldots b_{p-1} X_{n}(p) b_{p}\right)
$$

are bounded (as maps from $B^{p}$ to $B$ each with operator norm) uniformly in $n$. Assume that for each $n$, the algebras $\operatorname{Alg}\left(B, X_{j}(k): 1 \leq k \leq r\right), k=1, \ldots, n$, are free with amalgamation over $B$. Let

$$
Y_{n}(k)=\frac{1}{\sqrt{n}} \sum_{j=1}^{n} X_{j}(k), \quad 1 \leq k \leq r .
$$

Then as $n \rightarrow \infty$,

$$
\mu_{Y_{n}(1), \ldots, Y_{n}(r) ; B} \rightarrow \mu_{Z(1), \ldots, Z(r) ; B}
$$

weakly, where $Z(1), \ldots, Z(r)$ is a B-semicircular family with covariance $\eta$.

We can now state the result describing the asymptotics of the distributions of the $\Delta_{n}$-random variables $G_{n}(k)$. We consider $\Delta_{n}$ as a subalgebra of $L^{\infty}[0,1]$ consisting of functions that are constant on each of the intervals $\left[\frac{k}{n}, \frac{k+1}{n}\right], 0 \leq k<n$.

3.8. TheOREM ([16]). Let $\eta_{n_{i j}}: \Delta_{n} \rightarrow \Delta_{n}$ be defined by

$$
\eta_{n_{i j}}(d)=E_{n}\left(G_{n}(i) d G_{n}(j)\right) \text {. }
$$

Assume there exist maps $\eta_{i j}: L^{\infty}[0,1] \rightarrow L^{\infty}[0,1]$ such that whenever $d_{n} \in \Delta_{n}, d_{n} \rightarrow$ $d \in L^{\infty}[0,1]$ in norm, then also $\lim _{n} \eta_{n i j}\left(d_{n}\right)=\eta_{i j}(d)$. Then the family of $\Delta_{n}$-valued non-commutative random variables $G_{n}(1), \ldots, G_{n}(r)$ converges in $\Delta_{n}$-distribution to a family of $L^{\infty}[0,1]$ valued semicircular variables $Z(1), \ldots, Z(n)$ with covariance $\eta$, i.e., whenever $d_{n}(k) \in \Delta_{n}, 0 \leq k \leq p$ and $d_{n}(k) \rightarrow d(k) \in L^{\infty}[0,1]$ in norm, then

$$
\begin{gathered}
\lim _{n \rightarrow \infty} E_{n}\left(d_{n}(0) G_{n}\left(k_{1}\right) d_{n}(1) \ldots d_{n}(p-1) G_{n}\left(k_{p}\right) d_{n}(p)\right) \\
=E_{L^{\infty}[0,1]}\left(d(0) Z_{k_{1}} d(1) \ldots d(p-1) Z\left(k_{p}\right) d(p)\right) .
\end{gathered}
$$

Since $\eta_{n_{i j}}=0$ if $i \neq j$, from Theorem 3.8 and Theorem 3.6 we get an immediate corollary:

3.9. Corollary. The $\Delta_{n}$-valued random variables $G_{n}(1), \ldots, G_{n}(r)$ are asymptotically free with amalgamation over $L^{\infty}[0,1]$ as $n \rightarrow \infty$.

If $Z(1), \ldots, Z(r)$ are $B$-semicircular with covariance $\eta$, and $\phi: B \rightarrow \mathbf{C}$ is a state, it makes sense to ask what is the distribution of $Z(1), \ldots, Z(r)$ with respect to $\phi \circ E$. Using the Fock space model for the operators $Z(k)$, the remarks in $\S 3.3$ and Theorem 3.8 one gets: 
3.10. Corollary. Assume in the notation of Theorem 3.8 that $\eta_{i j}(1)=\delta_{i j} 1$, where 1 denotes the constant function 1 in $L^{\infty}[0,1]$. Then with respect to the functional $\phi_{n}$ (defined in §1.1) $G_{n}(1), \ldots, G_{n}(r)$ converges in (scalar-valued) distribution to a free semicircular family. In particular, the limit distribution of $G_{n}(k), 1 \leq k \leq r$ is the semicircular measure $\frac{1}{\pi}\left(1-t^{2}\right)^{1 / 2}$.

Combining Voiculescu's Theorem 2.1 and Theorem 3.8 in the case $\sigma=1$ identically, we get:

3.11. Corollary. Let $Z(1), \ldots, Z(r)$ be a family of $L^{\infty}[0,1]$-semicircular variables with covariance $\eta$. Let $\phi: L^{\infty}[0,1] \rightarrow \mathbf{C}$ be integration against Lebesgue measure. Consider the algebra $C^{*}\left(L^{\infty}[0,1], Z(k): 1 \leq k \leq r\right)$ as a (scalar-valued) non-commutative probability space with the functional $\phi \circ E_{L^{\infty}[0,1]}$. If $\eta_{i j}(a)=c_{i j} \phi(a), c_{i j} \in \mathbf{C}$, for all $a \in L^{\infty}[0,1]$, then $C^{*}(Z(1), \ldots, Z(r))$ are free from $L^{\infty}[0,1]$.

Motivated by this we proved:

3.12. THEOREM $([17,18])$. Let $Z(1), \ldots, Z(r)$ be a B-semicircular family with covariance $\eta$. If $\eta_{i j}(a)=c_{i j} \phi(a), c_{i j} \in \mathbf{C}$, for all $a \in B$. Then $C^{*}(Z(1), \ldots, Z(r))$ and $B$ are free in $C^{*}(B, Z(k): 1 \leq k \leq r)$ with respect to $\phi \circ E$.

4. Application: asymptotic distribution of $H_{n}$. Let $H_{n}=d_{n}+G_{n}(1)$ be as in $\S 1.3$, and assume that $d_{n} \rightarrow d \in L^{\infty}[0,1]$ in norm. Let $\eta_{n}: \Delta_{n} \rightarrow \Delta_{n}$ be given by

$$
\eta_{n}(d)=E_{n}\left(G_{n}(1) d G_{n}(1)\right)=\operatorname{diag}\left(y_{1}, \ldots, y_{n}\right), \quad d=\operatorname{diag}\left(z_{1}, \ldots, z_{n}\right) \in \Delta_{n},
$$

where

$$
y_{i}=\frac{1}{n} \sum_{j} \sigma_{n}^{2}(i, j ; 1) z_{j} .
$$

Assume that there exists a map $\eta: L^{\infty}[0,1] \rightarrow L^{\infty}[0,1]$, such that whenever $b_{n} \in \Delta_{n}$, $b_{n} \rightarrow b \in L^{\infty}[0,1]$ in norm as $n \rightarrow \infty, \eta_{n}\left(b_{n}\right) \rightarrow \eta(b)$ in norm.

Using Theorem 3.8, we deduce that $H_{n}$ converges in $\Delta_{n}$-valued distribution (in the sense explained in Theorem 3.6) to an $L^{\infty}[0,1]$-valued random variable $H=d+Z$, where $Z$ is an $L^{\infty}[0,1]$-semicircular variable with covariance $\eta$. Therefore, by the discussion in $\S 3.3$, we have that the distribution of $H_{n}$ with respect to $\phi_{n}$ converges as $n \rightarrow \infty$ to the distribution of $H$ with respect to $\phi=\psi \circ E_{L^{\infty}[0,1]}$, where $\psi$ denotes Lebesgue measure. It is therefore only necessary to compute the latter distribution.

The technical tool for this computation is the $R$-transform of Voiculescu $([26,25])$, later generalized for the many variable case by Speicher in [20]. We only consider the one-variable case here.

4.1. Let $X=X^{*}$ be a $B$-valued random variable, where $B$ is a $C^{*}$-algebra. Consider for $b \in B$ of small norm the power series

$$
G(b)=b+\sum_{k \geq 1} E\left(b(X b)^{k}\right) \in B
$$

Note that in the case $B \neq \mathbf{C}, G(b)$ contains less information than the distribution of $X$ (for example, it contains no information about $E\left(X b X b^{\prime} X\right)$ for $b, b^{\prime} \in B$ ). However, if $B$ 
has a linear functional $\phi$, and we are interested in the distribution of $X$ with respect to $\phi \circ E$, then it is enough to know $G(b)$, since

$$
C(z)=\phi(G(z 1))=z \phi\left(\left(1+\sum_{k \geq 1} E\left(X^{k}\right) z^{k}\right)\right)=z\left(1+\sum_{k \geq 1}(\phi \circ E)\left(X^{k}\right) z^{k}\right), \quad z \in \mathbf{C}
$$

contains all information about the moments of $X$.

4.2. Consider the function $R(b)$ defined for $b$ of small norm by the equation

$$
(G(b))^{-1}+R(G(b))=b .
$$

The function $R$ is called the $R$-transform of the distribution of $X$, written $R_{X}$.

4.3. TheOrem (Voiculescu, [25]). If B-valued random variables $X$ and $Y$ are free with amalgamation over $B$, then

$$
R_{X+Y}(b)=R_{X}(b)+R_{Y}(b), \quad b \in B .
$$

An analogous property in classical probability theory is possessed by the logarithm of the Fourier transform.

4.4. Using Theorem 4.3 and the discussion of $\S 4.1$, we can now give an equation for the distribution of $H$. Assume that $\eta$ is given by an integral kernel $\sigma^{2}$, i.e.,

$$
\eta(f)(x)=\int_{0}^{1} f(y) \sigma^{2}(x, y) d y .
$$

Note that because of (4.3), for sufficiently nice $\sigma_{n}(i, j ; 1), \sigma(x, y)=\lim _{n} \sigma_{n}([n x],[n y] ; 1)$, where $[x]$ denotes the integral part of $x$. Recall that $H=d+Z$. Since $d \in L^{\infty}[0,1], Z$ and $d$ are free with amalgamation over $L^{\infty}[0,1]$. Thus $R_{H}=R_{d}+R_{Z}$. It is easily seen that $R_{d}(b)=d$ for all $b \in L^{\infty}[0,1]$. By a computation in [25], we know that $R_{Z}(b)=\eta(b)$. Writing (4.6) for $G(b) \in L^{\infty}[0,1]$ gives for $x \in[0,1]$

$$
G(b)(x)=\left[b(x)-d(x)-\int_{0}^{1} \sigma^{2}(x, y) G(b)(y) d y\right]^{-1} .
$$

Let $a(z, x)=G(z 1)(x)$. Then we get

$$
a(z, x)=\left[z-d(x)-\int_{0}^{1} \sigma^{2}(x, y) a(z, y) d y\right]^{-1}
$$

so that $C$ of equation (4.5) is given by

$$
C(z)=\int_{0}^{1} a(z, x) d x
$$

which is the formula obtained by Girko and Casati in [4].

5. Applications to von Neumann algebras. Random matrix models have found many applications in the theory of free group factors (i.e., the von Neumann algebras generated in the left regular representation of free groups); see e.g. [26, 23, 6, 14, 15, 13]. The connection between Gaussian random band matrices and $L^{\infty}[0,1]$-valued semicircular families allows one to rephrase many of the theorems from the papers listed above 
in the language of operator-valued semicircular systems. This sometimes allows one to extend the theorems (with essentially the same proofs) to situations involving $B$-valued semicircular systems with arbitrary $B$ (we already saw an instance of this, passing from the $L^{\infty}[0,1]$-valued case in Corollary 3.11 to Theorem 3.12). We give a few examples.

5.1. Let $M$ be a $\mathrm{II}_{1}$ factor, i.e., a von Neumann algebra with a trivial center possessing a normal faithful trace $\tau$ with $\tau(1)=1$. If $p=p^{*}=p^{2}, q=q^{*}=q^{2}$ are two projections in $M$ with $\tau(p)=\tau(q)$, then there exists a unitary $u \in M, u^{*} u=u u^{*}=1$, such that $u p u^{*}=q$. Therefore, the algebra $p M p, p=p^{*}=p^{2} \in M$, depends only on $t=\tau(p)$ and is denoted $M_{t}$. The fundamental group $F(M)$ of $M$ is the subgroup of positive reals $\mathbf{R}_{+}$, generated by those $t$ for which $M_{t} \cong M$. This invariant of a $\mathrm{II}_{1}$ factor $M$ is very hard to compute.

We write $L(F(n))$ for the group von Neumann algebra of the free group with $n$ generators. The following theorem was obtained by Rădulescu using random matrix techniques:

5.2. Theorem (Rădulescu, [13]). $F(L(F(\infty)))=\mathbf{R}_{+}$.

Rephrasing the proof of Rădulescu in terms of operator-valued semicircular systems, we obtained the following, writing $*$ for the free product of two von Neumann algebras:

5.3. Theorem ([17]). Let $M$ be a $I I_{1}$ factor. Then $F(M) \subset F(M * L(F(\infty)))$.

5.4. Recall that a subfactor of a $\mathrm{II}_{1}$ factor $M$ (see $[8,7]$ ) is a unital inclusion of a $\mathrm{II}_{1}$ factor $N \subset M$. Given $N \subset M$, Jones in [8] assigned to the inclusion a number, called the index $[M: N]$. Rephrasing the random matrix techniques of Rădulescu [14] and using a construction of Popa [12], we obtained the following:

5.5. Theorem ([18]). Let $N \subset M$ be a finite-index finite depth inclusion of $I I_{1}$ factors. Let $P=M * L(F(n)), n$ integer or infinity. Then there exists a finite index finite depth subfactor $Q \subset P$, with $[P: Q]=[M: N]$.

Let

$$
I_{\mathrm{fd}}(M)=\{[M: N]: N \subset M \text { is a finite-depth subfactor }\} .
$$

This is an invariant of $M$, cf. [8]. Then we immediately get

5.6. Corollary. $I_{\mathrm{fd}}(M) \subset I_{\mathrm{fd}}(M * L(F(n)))$, for $n$ finite or an integer.

5.7. We have introduced in [19] a class of type III factors, called free Araki-Woods factors. Any type III factor can be written as a crossed product of a $\mathrm{II}_{\infty}$ von Neumann algebra by an action of $\mathbf{R}$. The $\mathrm{II}_{\infty}$ von Neumann algebra is canonically associated to the type III factor, and is called its core. We have shown

5.8. TheOrem ([17]). Let $M$ be a free Araki-Woods factor, and $N$ be its core. Then $N$ is generated by $L^{\infty}[0,1]$ and an $L^{\infty}[0,1]$-semicircular family $Z(k), k=1,2, \ldots$

Because of this, Gaussian random band matrices provide an intuitive picture for the core of a free Araki-Woods factor. This has led to a number of results about the cores and the free Araki-Woods factors themselves; we refer the reader to $[17,18]$. 


\section{References}

[1] S. R. Bahcall, Random matrix model for superconductors in a magnetic field, to appear in Phys. Rev. Letters.

[2] B. Blackadar, K-theory for operator algebras, Springer-Verlag, 1986.

[3] L. V. Bogachev, S. A. Molchanov and L. A. Pastur, On the density of states of random band matrices, Math. Notes 50 (1991), 1232-1242.

[4] G. Casati and V. Girko, Generalized Wigner law for band random matrices, Random Oper. and Stoch. Equ. 1 (1993), 279-286.

[5] G. Casati, V. Girko and L. Molinari, Equations for limit functions of band random matrices, Random Oper. and Stoch. Equ. 1 (1993), 91-94.

[6] K. Dykema, On certain free product factors via an extended matrix model, J. Funct. Anal. 112 (1993), 31-60.

[7] F. M. Goodman, R. de la Harpe and V. F. R. Jones, Coxeter graphs and towers of algebras, Springer-Verlag, 1989.

[8] V. F. R. Jones, Index for subfactors, Invent. math. 72 (1983), 1-25.

[9] M. L. Mehta, Random matrices, Academic Press, 1991.

[10] L. Pastur, Eigenvalue distribution of random operators and matrices, Séminaire Bourbaki, vol. 1991/92, No. 206, Exp. No. 758, 5, Astérisque, 1993, pp. 445-461.

[11] M. Pimsner, A class of $C^{*}$-algebras generalizing both Cuntz-Krieger algebras and crossed products by Z, in: D.-V. Voiculescu (ed.), Free Probability, Fields Institute Communications, vol. 12, American Mathematical Society, 1997, pp. 189-212.

[12] S. Popa, Markov traces on universal Jones algebras and subfactors of finite index, Invent. math. 111 (1993), 375-405.

[13] F. Rădulescu, $A$ one parameter group of automorphisms of $L\left(F_{\infty}\right) \otimes B(\mathcal{H})$ scaling the trace, C. R. Acad. Sci. Paris 314 (1992), 1027-1032.

[14] F. Rădulescu, Random matrices, amalgamated free products and subfactors of the von Neumann algebra of a free group, of noninteger index, Invent. math. 115 (1994), 347-389.

[15] F. Rădulescu, A type III $I_{\lambda}$ factor with core isomorphic to the von Neumann algebra of a free group, tensor $B(H)$, in: Recent advances in operator algebras (Orléans, 1992), no. 232, Astérisque, 1995, pp. 203-209.

[16] D. Shlyakhtenko, Random Gaussian band matrices and freeness with amalgamation, Internat. Math. Res. Notices 20 (1996), 1013-1026.

[17] D. Shlyakhtenko, Some applications of freeness with amalgamation, J. Reine Angew. Math. 500 (1998), 191-212.

[18] D. Shlyakhtenko, A-valued semicircular systems, preprint, 1997.

[19] D. Shlyakhtenko, Free quasi-free states, Pacific J. Math. 177 (1997), 329-368.

[20] R. Speicher, Combinatorial theory of the free product with amalgamation and operatorvalued free probability theory, Mem. Amer. Math. Soc. 132 (1998).

[21] D.-V. Voiculescu, Symmetries of some reduced free product $C^{*}$-algebras, in: Operator Algebras and Their Connections with Topology and Ergodic Theory, Lecture Notes in Mathematics, vol. 1132, Springer Verlag, 1985, pp. 556-588.

[22] D.-V. Voiculescu, Addition of certain non-commuting random variables, J. Funct. Anal. 17 (1986), 323-345.

[23] D.-V. Voiculescu, Free noncommutative random variables, random matrices and the $\mathrm{II}_{1}$ factors of free groups, in: Quantum probability and related topics, QP-PQ, VI, World Sci. Publishing, River Edge, NJ, 1991, pp. 473-487. 
[24] D.-V. Voiculescu, Limit laws for random matrices and free products, Invent. math. 104 (1991), 201-220.

[25] D.-V. Voiculescu, Operations on certain non-commutative operator-valued random variables, in: Recent advances in operator algebras (Orléans, 1992), no. 232, Astérisque, 1995, pp. 243-275.

[26] D.-V. Voiculescu, K. Dykema and A. Nica, Free random variables, CRM monograph series, vol. 1, American Mathematical Society, 1992. 